\title{
THE IMPERIAL CANCER RESEARCH FUND
}

$\mathrm{T}$ HE fifty-sixth annual report of the Imperial Cancer Research Fund*, for 1957-58, describes the lines of investigation in progress in the laboratories at Mill Hill and Lincoln's Inn Fields. They can be sub-divided as follows :

(1) Tumours induced by viruses.

Work continues on the virus ætiology of cancer by studying the mechanism whereby the virus gains access to the cell it infects, the virus's mode of replication and its specificity. Attempts are being made to grow the mouse leukæmia-inducing virus in tissue cultures of embryo fibroblasts, and by reciprocal transplantation experiments to find "whether the cellular elements in such transplants survive or whether virus is liberated to produce a new tumour by infection of the host's own cells".

(2) Tissue culture studies of tumour cells.

The reaction of tumour cells to environmental changes is being studied by observing the effect of variation in the tension of carbon dioxide on cells of the mouse sarcoma 37.

"The activities of individual cells in culture of the various mouse tumours are being examined by timelapse cinematography. $B P / 8 \mathrm{C}$ and $B A S / 56$ ascites tumour cells show 'tails' which may attach to the glass and which have great tensile strength and elasticity. The undulating surface membranes of these cells show very active pinocytosis and unusually large volumes of culture medium may be injected in this way."

(3) Preservation of tumours in the frozen state.

"The frozen tumour bank preserves tumours in an unaltered genetic state and these can therefore be used to check the respective inbred strains of mice for any genetic divergence which affects histocompatibility. In this way it has been shown, with a strain $A$ specific tumour, not only that $A_{2} G$ mice are genetically distinct from true $A$ but that the genetic change occurred prior to 1952."

(4) Heterotransplantation of human tumours.

Human sareoma tissue is being maintained by subcutaneous, intramuscular or intraperitoneal trans-

* Imperial Cancer Research Fund. Fifty-sixth Annual Report 1957-1958. Pp. 39. (London: Imperial Cancer Rezeareh Fund, 1959.) plantation in cortisone-treated hamsters, subcutaneously in cortisone-treated mice and in the anterior chamber of the eye in normal guinea pigs. The attempts to grow human lung cancer in guinea pig anterior chamber or in organ cultures were unsuccessful, but 25 per cent of human embryonic lung tissue grafts survived 22-49 days in mice treated with cortisone.

Other human embryonic tissues such as bone and bowel, some human adult tissue, for example, skin and transitional epithelium, and human prostatic carcinoma and adult rodent lung and prostate have all been maintained in fluid media. "Organ cultures of mouse prostate have been employed to study the direct action of ostrogens and androgens on glands from mice of different ages. The cestrogens produced epithelial atrophy and testosterone a stimulation."

(5) Hormone-dependent breast cancer.

About 50 per cent of human breast cancers cease to proliferate if they are totally deprived of the hormones which control cell multiplication in the normal breast. At present the operative technique of cutting off the supply of hormones is by the surgical removal of the ovaries, both adrenals and the pituitary. This somewhat drastic treatment may give spectacular growth regression and clinical improvement, but unfortunately these have proved to be temporary.

(6) A pregnancy-dependent mouse tumour.

The spontaneous mammary tumour BR6 has the peculiarity of always first appearing during pregnancy and mostly regressing after parturition. The tumour incidence is very high (in excess of 97 per cent in more than 400 mice that lived longer than six months, had more than two litters, and where the mothers had developed tumours). Under certain conditions some females not only remain tumour-free but give rise to tumour-free sub-lines which ". . . appear from transplantation experiments, to be genetically different from the tumour-prone line and one aspect of this genetic difference may be a greater androgen production by the males of the tumour-prone lines".

I. H.IEGER

\section{BEHAVIOUR OF SEA URCHINS}

$\mathrm{M}$ ANY of the habits of sea urchins were observed by A. N. Sinclair, during day and night diving in the waters around Sydney with members of the Underwater Research Group of New South Wales. An aqualung and a waterproof torch were used (Austral. Mus. Mag., 13, No. 1 ; March 15, 1959).

When diving in daylight Sinclair was impressed by the numbers of the large, rough-spined sea urchin Centrostephanus rodgersii. Many of these were seen in hollows carved in the sandstone rocks, and often the hollows were deep enough to contain the whole urchin, but were never as deep, comparatively, as the sharply etched hollows carved by the smaller urchins, Heliocidaris erythrogramma. Other urchins appeared to be motionless, with the spines sticking out at right angles to the body surface in a typical 'hedgehog', or defence, position.

It was found that after darkness set in the urchins became active. Within an hour or two of sunset, numbers of Centrostephanus were seen 'out walking' on the rocks, but they did not seem to favour walking on the sand. The spines at such times were generally arranged in groups or cones. If a torch was shone on the urchin for a short time no reaction to the light could be observed, but if the urchin was touched it immediately assumed the 'hedgehog' position seen in daylight.

Results of marking specimens of $C$. rodgersii in Clovelly Bay, Sydney, had shown that the urchins moved up to 3 or $4 \mathrm{ft}$. from their rock holes within 